\title{
BMJ Open Hydroxychloroquine for prevention of recurrent miscarriage: study protocol for a multicentre randomised placebo- controlled trial BBQ study
}

\author{
Elisabeth Pasquier, ${ }^{1}$ Luc de Saint-Martin, ${ }^{2}$ Gisèle Marhic, ${ }^{3}$ Celine Chauleur, ${ }^{4}$ \\ Caroline Bohec, ${ }^{5}$ Florence Bretelle, ${ }^{6}$ Véronique Lejeune-Saada, ${ }^{7}$ \\ Jacob Hannigsberg, ${ }^{8}$ Geneviève Plu-Bureau, ${ }^{9}$ Virginie Cogulet, ${ }^{10}$ Philippe Merviel, ${ }^{8}$ \\ Dominique Mottier ${ }^{11}$
}

To cite: Pasquier E, de Saint-Martin L, Marhic G, et al. Hydroxychloroquine for prevention of recurrent miscarriage: study protocol for a multicentre randomised placebo-controlled trial BBQ study. BMJ Open 2019;9:e025649. doi:10.1136/ bmjopen-2018-025649

- Prepublication history and additional material for this paper are available online. To view these files, please visit the journal online (http://dx.doi. org/10.1136/bmjopen-2018025649).

Received 2 August 2018 Revised 11 February 2019 Accepted 12 February 2019

Check for updates

(C) Author(s) (or their employer(s)) 2019. Re-use permitted under CC BY-NC. No commercial re-use. See rights and permissions. Published by BMJ.

For numbered affiliations see end of article.

Correspondence to

Dr Elisabeth Pasquier;

elisabeth.pasquier@chu-brest.fr

\section{ABSTRACT}

Introduction Recurrent miscarriage (RM), defined by three or more consecutive losses during the first trimester of pregnancy, affects $1 \%-2 \%$ of fertile couples. Standard investigations fail to reveal any apparent cause in $50 \%$ of couples. However, on the basis of animal models and clinical studies, several hypotheses have been put forward concerning underlying mechanisms of RM: altered ovarian reserve, progesterone defect, thrombotic and/or endothelial dysfunction and immunological disturbances. Nonetheless, no study has yet reached conclusive beneficial clinical evidence for a potential treatment in unexplained RM. Hydroxychloroquine ( $\mathrm{HCQ}$ ) is a molecule with extensive safety data during pregnancy. The pharmacological properties of HCQ (eg, antithrombotic, vascular protective, immunomodulatory, improved glucose tolerance, lipidlowering and anti-infectious) could be effective against some mechanisms of unexplained RM. Furthermore, eventhough clinical benefit of HCQ is suggested in prevention of thrombotic and late obstetric events in antiphospholipid (APL) syndrome, there are no data suggesting the benefit of $\mathrm{HCQ}$ in RM in the presence of APL antibodies.

Methods and analysis Taken all together and given the low cost of $\mathrm{HCQ}$, the aim of this multicentre, randomised, placebo-controlled, double-blind study is to investigate whether HCQ would improve the live birth rate in women with RM, irrespective of maternal thrombophilic status: (1) no known thrombophilia, (2) inherited thrombophilia or (3) APL antibodies. The primary end point is a live and viable birth. After confirming eligibility and obtaining consent, 300 non-pregnant women will be randomised into two parallel groups for a daily oral treatment (HCQ $400 \mathrm{mg}$ or placebo), initiated before conception and stopped at 10 weeks' gestation. If pregnancy does not occur after 1 year, the treatment will be stopped.

Ethics and dissemination Agreement from the French National Public Health and Drug Security Agency (160765A-22) and ethical approval from the Committee for the Protection of Persons of NORD-OUEST I (2016001330-97) have been obtained.

Trial registration numbers NCT0316513; Pre-results.

\section{Strengths and limitations of this study}

- Despite numerous fundamental research publications and clinical trials, the only recommendation that applies to follow-up of childbearing women suffering from recurrent miscarriage (RM) relies on cocooning.

- This is the first randomised placebo-controlled study that aims to investigate whether oral hydroxychloroquine (HCQ) would improve the live birth rate in women with RM irrespective of maternal thrombophilic status.

- As a possible limitation, this study is based on the hypothesis that RM often results from combined failures which could benefit from the pleiotropic effects of $\mathrm{HCQ}$.

- As HCQ should probably be administrated at least two menstrual cycles before conception to have an effect, women will be advised not to get pregnant during this period, but we do not plan to exclude those who will conceive too early.

\section{INTRODUCTION}

\section{Background}

Recurrent miscarriage (RM) is a common public health reproductive burden causing both physical and emotional distress. To date, there is no treatment whose benefit has been clearly proved, even in the presence of well-known risk factors of RM. Most importantly, the absence of benefit has been clearly demonstrated for some treatments that are yet empirically proposed.

\section{Unexplained RM}

A sporadic miscarriage is clinically detected in approximately $10 \%-15 \%$ of pregnancies. Fetal development usually stops before 10 weeks. ${ }^{1}$ Given the RM frequency among fertile couples $(1 \%-2 \%)$ being significantly higher than the expected random one 
$(15 * 15 * 15 \%=0.34 \%)$, RM is most often defined as three or more consecutive losses. Apart from the detection of a lethal chromosomal abnormality on products of conception, the underlying mechanism of loss remains unknown in most cases. The rate of normal embryonic karyotypes in RM steadily increases from the third loss, suggesting alternative mechanisms than meiotic aberrations. ${ }^{2}$ Standard investigations fail to reveal any apparent cause in $\sim 50 \%$ of the women. However, on the basis of animal models and clinical studies, several hypotheses have been put forward. Here, we focus on thrombosis and both endothelial and immune dysfunctions. Those could be targeted by pharmacological properties of hydroxychloroquine (HCQ).

An association with some inherited thrombophilia has been reported (factor V Leiden, mutation G20210A of the prothrombin and protein $\mathrm{S}$ deficiency), although the OR was most often $\leq 2 .{ }^{3}$ Animal models have demonstrated that some actors of the haemostatic system may participate in normal implantation and placental development regardless of the coagulation process. ${ }^{45}$ In humans, a basal prothrombotic state outside of pregnancy has been measured in women with previous RM and without known thrombophilia. $^{6-8}$ This relative prothrombotic state, measured at distance of any obstetrical event, could reflect chronic endothelium damage in those women. ${ }^{910}$ Notwithstanding, the clinical trials that have assessed antithrombotic treatments (aspirin initiated before or after conception, eventually combined with low molecular weight heparin [LMWH], or LMWH alone initiated after pregnancy diagnosis) did not show any benefit in prevention of further loss. ${ }^{11-14}$ Likewise, no benefit of LMWH has been shown in the subgroup of women with inherited thrombophilia (TIPPS study). ${ }^{15}$ However, we emphasise that the subcutaneous route of LMWH administration does not allow assessing this treatment at the critical time of implantation onset in fertile women. Indeed, the injections cannot be routinely initiated before 5 weeks' gestation.

Regarding immune dysfunction, apart from the detection of many auto-antibodies (antiphospholipid [APL], antithyroid), ${ }^{16}$ attention is now focused on natural killer cells, whose temporal and spatial distribution in the uterine mucosa suggests that they contribute to control trophoblast invasion and cytokine response at the maternal-fetal interface (balances Th-1/Th-2 and Th-17/Treg). Th-2 and T-reg preponderance in normal pregnancy shifts to Th-1 and Th-17 predominance in RM. ${ }^{17}$ Otherwise, an overexpression of Toll receptors in decidua was observed in women with RM, especially TLR4 and TLR9 receptors. ${ }^{19}$ Immunomodulatory treatments have therefore been proposed and assessed without conclusive results (paternal leucocyte immunisation, ${ }^{20}$ intravenous immunoglobulins ${ }^{21}$ and small doses of corticosteroids). ${ }^{22}$

Despite numerous fundamental research publications and clinical trials, the only current recommendation for follow-up of childbearing women suffering from unexplained RM relies on cocooning. ${ }^{1723}$

\section{RM in the presence of APL antibodies}

Antiphospholipid syndrome (APS) is diagnosed in the presence of persistent, above the 99th percentile, APL levels and of at least one clinical manifestation (obstetrical, arterial or venous thrombosis). 'Three or more unexplained consecutive spontaneous abortions before the 10th week of gestation, with maternal anatomic or hormonal abnormalities and paternal and maternal chromosomal causes excluded ${ }^{24}$ delineates one of the possible manifestations of APS. Even though RM is one accepted clinical manifestation of APS, its specificity is low, when compared with the occurrence of fetal death after 10 weeks of amenorrhoea. ${ }^{24}$ The benefit of low-dose aspirin and/or LMWH or heparins for secondary prevention of RM has not been demonstrated in women who present with APL antibodies and no other clinical manifestation of APS. ${ }^{25}$ The HEPASA study found no benefit of combination therapy of LMWH and low-dose aspirin in women who presented with APL, without arterial or venous thrombosis history, and who had at least two fetal losses before 32 weeks of amenorrhoea. ${ }^{25}$ As a consequence, no clinical evidence has been drawn for an optimal management of these women.

\section{Rationale for hydroxychloroquine}

Chloroquine and HCQ are used for centuries to treat malaria. Because of a more favourable usage profile and immunomodulatory properties, HCQ use became common for the treatment of autoimmune and inflammatory diseases. Other therapeutic properties of HCQ have since been discovered, or rather rediscovered, and assessed in a more consistent manner. The following HCQ properties could be effective against mechanisms of RM.

Since 30 years, benefit of HCQ to prevent venous thromboembolism (VTE) has been suspected ${ }^{26-35}$ (1) among patients with SLE $^{26} 31$ and (2) among all-comer patients, free of autoimmune disease, after orthopaedic surgery. ${ }^{27-30}$ Thus, before the use of LMWH, HCQ was assessed in the 1970s in the prevention of VTE events after orthopaedic surgery. The results of randomised trials, ${ }^{28-30}$ (around 10000 patients included) led to the conclusion that HCQ was beneficial in preventing VTE after orthopaedic surgery. ${ }^{28}$ In patients with SLE, in the presence or absence of APL antibodies, studies have suggested the benefit of HCQ for the primary prevention of both arterial and venous thrombotic events. ${ }^{26} 31-33$ In primary APS, HCQ reduced the thrombus size after vessel injury in mice ${ }^{34}$ and would improve the secondary prevention of VTE in humans, in comparison to oral anticoagulants used alone. ${ }^{35}$ The antithrombotic action of HCQ would be inherent to some biological effects (in the absence $^{36-38}$ or in the presence ${ }^{39} 40$ of APL antibodies). Otherwise, much data support an endothelium protective action of HCQ via antidiabetic, ${ }^{41}$ lipidlowering, ${ }^{42}$ antioxidant ${ }^{43}$ effects or a direct endothelial protection, via ERK5 protein kinase activation. ${ }^{44}$

Regarding immunomodulation, HCQ has an impact on the innate immunity by inhibition of the activation 
of some Toll receptors $(3,7,9) .{ }^{45-47}$ HCQ decreases the circulating levels of interleukin $1,2,{ }^{48} 6,{ }^{49} \mathrm{TNF}^{49} 50$ and interferon- $\gamma,{ }^{49}$ promoting the TH2 processes that prevail in a "normal pregnancy". Otherwise, HCQ decreases APL plasma levels ${ }^{51}$ and interferes with both endothelial cell activation and TNF- $\alpha$ production, two major key pathways involved in APS. ${ }^{52-54}$

We also outline the well-known anti-infectious action of HCQ that originates from the alkalinisation of intracellular acidic vesicles and might inhibit the growth of intracellular microorganisms. This could act against chronic endometritis, an additional mechanism suspected in RM.

Remarkably, the use of HCQ (and closely related molecules) as anti-malarial or in auto-immune diseases during pregnancy ${ }^{5-57}$ and lactation ${ }^{56}$ has provided extensive safety data during pregnancy and even during breast feeding.

Otherwise, oral administration of HCQ facilitates its prescription early, before conception, thus enabling fetal exposure from the very beginning of time period at risk for activation of underlying mechanisms of RM. Noteworthy, HCQ has never been evaluated in the indication of RM with or without APL antibodies.

Taken all together and given that RM is a stereotyped clinical entity whatever the maternal thrombophilic status, we aimed at evaluating the role of HCQ in prevention of RM, with or without APL antibodies or inherited thrombophilia. We therefore initiated a multicenter placebo-controlled trial sponsored by Brest University Hospital and supported by a grant from the French Ministry of Health (PHRCN-17-0573).

\section{STUDY OBJECTIVES \\ Primary objective}

The aim of this placebo-controlled, double-blind trial is to investigate whether HCQ (orally administrated before conception until 10 weeks of gestational age) would improve the live birth rate of $15 \%$ or more, in women with previous RM, irrespective of their biological thrombophilic status.

\section{Secondary objectives}

We aim to investigate whether

- HCQ effect would be different among women with inherited thrombophilia or APL antibodies.

- HCQ would have an impact on the occurrence of some pregnancy events (early miscarriage, intrauterine fetal death, placental vascular disease).

- The impact of HCQ would be different in subgroups of women at higher or lower risk of recurrence according to the number of previous miscarriages $(\geq 3)$, the maternal age ( $\geq 35$ or $<35$ years), the parity: previous live birth, a previous late fetal death after 10 weeks' gestation.

We aim to confirm that HCQ has no negative impact on the chance of getting pregnant, the newborn (gestational age, birth weight, survival at 28 days, congenital abnormalities), the child at 6 months.

\section{METHODS}

\section{Study design}

This is a prospective, randomised 1:1 placebo-controlled, double-blind, and French multicentre trial of phase III (with two parallel-groups [HCQ $400 \mathrm{mg}$ or placebo]). At randomisation, the minimisation method will be used to balance between the two groups and two main determinants of miscarriage recurrence: maternal age ( $\leq 35$ or $>35$ years) and number of previous losses ( 3 or $\geq 4)$.

\section{Study setting}

Women are currently being enrolled in university hospitals (gynaecology units: Besançon, Brest, Clermont Ferrand, Lille, Marseille, Nantes, Paris Cochin, Paris Bichat, Rennes, Saint-Etienne; internal medicine units: Brest, Paris Saint-Antoine) or in general hospitals (gynaecology units: Auch, Quimper, Mont de Marsan, $\mathrm{Pau})$.

\section{Population}

The study population consists of women trying to conceive and who had experienced at least three consecutive miscarriages at the first trimester of pregnancy (normal parental karyotypes, no uterine cavity abnormality that might explain the losses). Through medical meetings, emails and letters, all obstetricians and internal medicine practitioners working in each participating centre's catchment area have been informed and trained on BaBy hydroxychloroQuine study. All of those are asked to refer potentially eligible women to the unit participating in the study for screening. In each centre, patient recruitment is ensured by already in place settings such as specific RM consultations or other OBS/GYN patient management units. In addition to this recruitment approach, poster information in consultation waiting rooms is being used to reach out to more patients.

At the inclusion visit, the medical investigator checks inclusion and exclusion criteria of eligible women.

\section{Inclusion criteria}

- Women aged between 18 and 37 years inclusive.

- Women trying to conceive.

- Women with at least three previous consecutive miscarriages in the first trimester of pregnancy of unknown origin defined as

- Normal parental karyotypes.

- No uterine cavity abnormality that might explain RM (ultrasound scan, hysteroscopy or hysterography).

- In case of persistent positive APL antibodies according to the biological criteria of Myakis, ${ }^{24}$ no previous thrombotic or obstetrical event defined in 
APS, ${ }^{24}$ except for RM in the first trimester of pregnancy .

- Women who have given their informed consent.

\section{Exclusion criteria}

- Ongoing pregnancy.

- Normal pregnancy (live and viable birth) since the last miscarriage.

- Abnormal parental karyotype.

- Uterine cavity abnormality that might explain RM in the first trimester of pregnancy.

- APS defined as both

- Persistent positive APL antibodies: lupus anticoagulant and/or APL (anticardiolipin or anti béta2 GPI, IgG or IgM) titers $>99$ th percentile or $>40$ with at least 12 weeks interval between two positive determinations (persistent antibodies).

- A specific clinical setting of APS (thrombotic or obstetrical, apart from RM in the first trimester of pregnancy) according to Myakis criteria. ${ }^{24}$

- Known contraindication to a treatment by HCQ (retinopathy, hypersensitivity to chloroquine or HCQ, G6PD deficiency, acute intermittent porphyria, chronic liver or kidney insufficiency, extensive cutaneous psoriasis not controlled by local treatment, significant chronic digestive or haematologic disease) or known rare disorder of lactose metabolism (excipient).

- Past history of epilepsia or psychotic disorders.

- Indication to a treatment by HCQ according to the marketing authorisation (MA) (rheumatoid arthritis, Lupus, solar eczema).

- Previous exposure for $>4$ years to chloroquine or HCQ.

- Previous inclusion in this study.

- Woman unable to consent, protected under the terms of the law, or woman deprived of liberty by judicial or administrative decision.

- Non affiliation to the social security system.

- Impossible to follow-up.

\section{Allocation, randomisation and blinding}

After inclusion and obtaining written consent, an identification number is assigned to the patient through a secure server providing access to electronic case report forms generated by the CIC Brest (via the 'Capture System' software). The randomisation is done using the Capture Systemsoftware with implementation of a randomisation with minimisation to balance between the two treatment groups according to the main determinants of miscarriage recurrence: age ( $\leq 35$ or $>35$ years) and number of miscarriages $(3$ or $\geq 4)$.

The patient, the investigator and all medical and paramedic professionals taking care of the patient will be blinded to the treatment group. The packaging of placebo and HCQ will be provided in the form of capsules with identical external shape and packaging. Information on the correspondence between treatment group and the patient number will be held by both the central pharmacy of Brest CHRU and the Data Management Unit.

\section{Intervention}

Included patients are assigned to receive either $400 \mathrm{mg}$ of oral HCQ per day, administered in two divided doses of $200 \mathrm{mg}$ or two daily doses of oral placebo. Thus, treatment is initiated before conception and stopped at 10 weeks' gestation (weeks of amenorrhoea) or after 1 year of treatment in the absence of pregnancy.

\section{Rationale for choosing oral $\mathbf{4 0 0} \mathbf{m g}$ daily HCQ}

It is the usual route of administration and dose for indications specified by the MA. This choice of dose comes from the evaluation of the risk-benefit balance in the MA indications and, over all, from the fact that data of good tolerance have been compiled to this (usual) dosage in literature. Otherwise, in most cases, fetal development stops before 10 weeks of amenorrhoea. Treatment exposure is essential from the very beginning of implantation, a developmental stage that can potentially be disturbed by the mechanisms responsible for miscarriage. The oral administration allows starting the treatment before conception. This drug has a long half-life (from 30 to 60 days). Its full effect is obtained only after certain duration of exposure. That is why its administration is required before conception and its discontinuation after 10 weeks of amenorrhoea, that is, before the end of the first trimester of pregnancy which is at the highest risk for RM.

\section{Treatments frequently given in combination}

- Folic acid and other vitamin supplements.

- Aspirin at low dose, which could be given by some investigators to prevent the recurrence of vasculo-placental disease (preeclampsia, intra-utero growth restriction) or as part of a primary prevention of vasculo-placental events in women with high levels of persistent APL antibodies.

\section{Study plan and procedures}

Before pregnancy, women are monthly contacted by phone. The non-pregnant women are systematically seen in consultation after 6 and 12 months of treatment. In the absence of pregnancy after 1 year, the treatment will be withdrawn and patients will be contacted by phone at 14 and 18 months (end-of-study visit).

During pregnancy, women are seen monthly in consultation (first 4 months) or contacted by phone. The treatment is withdrawn at 10 weeks' gestation or earlier in case of pregnancy loss.

After delivery, the women are seen in consultation at $\sim 2$ months and contacted by phone at 6 months (end-ofstudy visit).

Four tubes with $2.7 \mathrm{~mL}$ sodium citrate and four tubes with $5 \mathrm{~mL}$ SST (for plasma and serum bank, respectively) are collected at both the inclusion visit and the visit of pregnancy onset or the visit planned after 1 year of treatment in the absence of pregnancy. Additionally, 
two tubes of $5 \mathrm{~mL}$ EDTA are collected for DNA bank at inclusion. The biological collection of plasma is carried out within 3 hours after receipt of tubes. DNA extraction is carried out within 1 week of collection (and at the latest within a month if the sample is frozen at $-80^{\circ} \mathrm{C}$ pending extraction). Those specimens are designed to identify prognostic factors (including thrombophilic work-up) for efficacy of the study treatment.

\section{End points}

The primary end point is a live and viable birth.

The secondary end points are as follows:

- A live and viable birth, for the subgroup analyses.

- The occurrence of miscarriage, in-utero fetal death, placental vascular disease and premature delivery.

- Gestational age at miscarriage.

- Concerning the child: gestational age and weight at birth, survival at 28 days, safety data at 6 months of life and congenital abnormality.

\section{Safety considerations, safety monitoring and AE reporting}

Apart from adverse events (AE) and serious adverse events (SAE), some safety parameters will be systematically recovered: $\mathrm{CBC}$, visual and neurological symptoms for women and reports of ultrasound and post-natal medical visits for children. AE are defined as any untoward medical occurrence in a person who consents to biomedical research whether this event is related or not to the research or experimental drug on which this research addresses. The gravity of SAE is defined by one of the following findings: death, life-threatening situation, significant or sustained incapacity or disability, hospitalisation, prolongation of hospitalisation, deformity or birth defect and potentially serious event. Any SAE or event of interest, regardless of its causal relationship with the trial treatment or the research, must be reported to the sponsor, as soon as possible and the latest 24 hours after their occurrence for SAE. The investigator is in charge of noting and reporting all SAE occurring during the study, from the date of the signed informed consent, during the study's expected duration of follow-up (6 months after the treatment cessation for women and at age 6 months for newborns). Furthermore, regardless of the event occurrence deadline after the end of the study, all SAE that may be due to the research must be declared to the sponsor when there is no other cause and effect accounted for (ie, serious events that may occur long after exposure to the drug, such as cancers or congenital abnormalities).

Some AE and SAE are expected and can be linked to 1) pregnancy, (2) the disease (occurrence of another miscarriage) or (3) study treatment (benign, adverse reaction such as digestive symptoms or severe, serious adverse reaction such as skin reaction, which requires to stop the treatment immediately).

\section{Independent Data and Safety Monitoring Board}

The Independent Data and Safety Monitoring Board (IDSMB) comprises eight members without competing interests, not directly involved in other aspects of the trial and independent from the sponsor: seven voting members, experts in complementary fields of the pathology and clinical trials (internal medicine, methodology, specialist of teratogenic agents, neonatology, haematology and ophthalmology) and a non-voting independent statistician. IDSMB monitors the data that affect patient safety, provide independent expertise for the evaluation of $\mathrm{AE}$ occurring during the study, express an opinion concerning the benefit-risk ratio and provide recommendations in order to help the steering committee to take decisions on protocol modification or early termination of the study. The members of the IDSMB will have to meet every 20 inclusions. The IDSMB will transmit its recommendations to the steering Committee, which decides whether or not to stop the study. Given the safety data and the treatment benefit, several different recommendations can be provided by the IDSMB: (1) continuation of study without protocol modification, (2) continuation of study with protocol modification, (3) temporary discontinuation of inclusions and (4) early termination of the study.

Over the course of study, the IDSMB will have an advisory role in reassessing the benefit-risk ratio of the study.

\section{Number of patients}

The total number of scheduled patients to be recruited is 300, on the basis of the following hypotheses:

- $80 \%$ of the included women should get pregnant (ALIFE study). ${ }^{11}$

- Among women who will get pregnant, we suppose that the rate of achieved pregnancy will be of $70 \%$ in the placebo group (PREFIX study). ${ }^{14}$

To demonstrate a minimal clinically important difference of $15 \%$ in live birth rates on HCQ (ie, a $50 \%$ relative risk reduction in pregnancy loss), we need to enrol 300 women for a power of $80 \%$, and a two-tailed alpha risk of $5 \%$.

The anticipated duration of recruitment is 3 years, and the duration of participation of each patient is from 7 to 29 months.

\section{Analyses plan}

Analyses will be first conducted on an intention-to-treat basis for all women who have started pregnancy. Hierarchical analysis including comparison of the number of live and viable births, between the two treatment groups (1) among all the women, (2) among women without known APL antibodies and (3) among women without known thrombophilia (APL or inherited thrombophilia) is planned. We plan to adjust for age and number of previous losses if the difference between the two treatment groups is statistically significant $(\mathrm{p}<0.05)$. 
Secondary analyses will be conducted on an intentionto-treat mode:

For all included women, comparison of the number of live and viable births between the two treatment groups.

Among women who will get pregnant,

- Measurement of treatment effect in women at higher risk of further miscarriage, according to the presence of thrombophilia, having history of intrauterine fetal death after 10 weeks' gestation, having history of miscarriage, more than three previous miscarriage, or no previous newborn baby.

- Comparison between the two treatment groups of the occurrence of several obstetrical events (miscarriage and intrauterine fetal death, vasculo-placental disease, premature birth).

- Comparison between the two treatment groups of newborn clinical data (gestation time at delivery, birth weight adjusted to the gestation time, number of newborn babies living at 28 days and congenital abnormalities).

A 'per-protocol analysis' (primary end point) will include only patients properly exposed to the study treatment, that is, (1) at least during a whole menstrual cycle before pregnancy onset and until 10 weeks of amenorrhoea (2) compliance $\geq 80 \%$.

No unblinded interim analysis is planned.

The biological collection will be carried out by the CRB of the CHRU of BREST and integrated into the collection 'thrombosis' declared to the Ministry (2008-214/ DC-2009-925).

\section{DISCUSSION}

To date, there has not yet been an optimal therapy with conclusive clinical benefit for unexplained RM, in the absence or presence of inherited thrombophilia. Providing some psychological support seems to be the only available therapy for women with unexplained RM. Although the probability of a further normal pregnancy seems high $(\sim 70 \%$ at age 32 years after three consecutive miscarriages) $)^{58}$ the proposed therapeutic interventions are sometimes excessive (eg, possible side effects) and costly (eg, intravenous immunoglobulins and assisted procreation). Therefore, it is of utmost importance to investigate other therapeutic options.

In RM, women with high titers of APL antibodies but without any other previous clinical event listed in APS (thrombotic or obstetric event), benefit of antithrombotic treatment (aspirin or LMWH) remains controversial. Clinical benefit of HCQ has not been demonstrated in prospective studies with robust methodology. ${ }^{59}$

In conclusion, the benefit-risk ratio and low cost are strong incentives for assessment of HCQ in unexplained RM irrespective of maternal thrombophilic status.

\section{Ethics and dissemination}

The necessary trial insurance is provided by HDI Gerling Industrie Versicherung (PARIS) Protocol version V3.0,
30 November 2017. Dissemination plan: We plan to publish study results in an international peer-reviewed journal. Negative as well as positive results will be published. Authors will have to meet the principles of the Vancouver Declaration. The results will be presented at both national and international conferences of relevance. A letter will inform the participants about the study results when requested. Furthermore, the results will be presented at all involved hospitals and participating wards.

\section{Author affiliations}

${ }^{1}$ EA 3878, Brest University Hospital, Brest, France

${ }^{2}$ EA 3878 Groupe d'Etude de la Thrombose de Bretagne Occidentale, Brest, France

${ }^{3}$ Centre d'Investigation Clinique-INSERM 1412, Brest, France

${ }^{4}$ Thrombosis Research Group, University Hospital Bellevue, Saint-Etienne, France

${ }^{5}$ Division of Gynaecology, Hôpital François Mitterand, Pau, France

${ }^{6}$ Division of Gynaecology, Université de la Méditerranée, Hôpital Nord, Marseille,

France

${ }^{7}$ Division of Gynaecology, Hôpital d'Auch, Auch, France

${ }^{8}$ Division of Gynecology, Brest University Hospital, Brest, France

${ }^{9}$ Division of Gynaecology, APHP, Hôpital Cochin, Paris, France

${ }^{10}$ Department of Pharmacy, Brest University Hospital, Brest, France

${ }^{11}$ Brest University Hospital, Brest, France

Acknowledgements The authors thank the clinical network Gynobs29 of the gynecologists and obstetricians of West Brittany and the national research network GOCIC "Réseau Gynécologie Obstétricale des Centres d'Investigation Clinique".

They also thank Zarrin Alavi (INSERM, CIC 1412) for proofreading and all the other investigators of the study: Dr Tiphaine Raia-Barjat (Gynecology, Saint-Etienne), Dr Nathalie Lesavre (Centre d'Investigation Clinique, Hôpital Nord, Marseille), Arsène Mékinian (APHP, Hospital Saint-Antoine, Paris), Norbert Winer (Gynecology, Nantes), Charles Bellot and Gaelle Le Foll (Gynecology, Quimper), Céline Pimentel (Gynecology, Rennes), Aude Bourtembourg (Gynecology, Besançon), Véronique Debarge (Gynecology, Lille), and Dominique Luton (APHP, Bichat).

Contributors EP conceived and designed the study. EP drafted the original grant proposal and trial protocol. LDS-M provides methodological and statistical expertise. GM has assisted in developing the protocol, helped with implementation and has responsibilities for day-to-day running of the trial including participant recruitment, data collection and liaising with other sites. JH and PM have helped with protocol implementation. CB, CC, FB, VL-S, GP-B and DM have participated in the design of the study. VC coordinates treatment production and dispensation. All authors critically reviewed and approved the final version of the manuscript.

Funding The study was supported by a grant from the French Ministry of Health (PHRCN-17-0573).

Competing interests None declared.

Patient consent for publication Obtained.

Ethics approval Agreement from the French National Public Health and Drug Security Agency (160765A-22) and ethical approval from the Committee for the Protection of Persons of NORD-OUEST I (2016-001330-97) have been obtained on 4 November 2016 and 2 March 2017, respectively.

Provenance and peer review Not commissioned; externally peer reviewed.

Open access This is an open access article distributed in accordance with the Creative Commons Attribution Non Commercial (CC BY-NC 4.0) license, which permits others to distribute, remix, adapt, build upon this work non-commercially, and license their derivative works on different terms, provided the original work is properly cited, appropriate credit is given, any changes made indicated, and the use is non-commercial. See: http://creativecommons.org/licenses/by-nc/4.0/.

\section{REFERENCES}

1. Branch DW, Gibson M, Silver RM. Clinical practice. Recurrent miscarriage. N Engl J Med 2010;363:1740-7.

2. Ogasawara M, Aoki K, Okada S, et al. Embryonic karyotype of abortuses in relation to the number of previous miscarriages. Fertil Steril 2000;73:300-4. 
3. Rey E, Kahn SR, David M, et al. Thrombophilic disorders and fetal loss: a meta-analysis. Lancet 2003;361:901-8.

4. Isermann B, Sood R, Pawlinski R, et al. The thrombomodulin-protein C system is essential for the maintenance of pregnancy, Nat. Med 2003;9:331-7.

5. Li W, Zheng X, Gu JM, et al. Extraembryonic expression of EPCR is essential for embryonic viability. Blood 2005;106:2716-22.

6. Laude I, Rongières-Bertrand C, Boyer-Neumann C, et al. Circulating procoagulant microparticles in women with unexplained pregnancy loss: a new insight. Thromb Haemost 2001;85:18-21.

7. Rai R, Tuddenham E, Backos M, et al. Thromboelastography, whole-blood haemostasis and recurrent miscarriage. Hum Reprod 2003;18:2540-3.

8. de Saint Martin L, Duchemin J, Bohec C, et al. Increased thrombin generation measured in the presence of thrombomodulin in women with early pregnancy loss. Fertil Steril 2011;95:1813-5.

9. Pasquier E, De Saint Martin L, Bohec C, et al. Unexplained pregnancy loss: a marker of basal endothelial dysfunction? Fertil Steril 2013;100:1013-7.

10. Kharazmi E, Dossus L, Rohrmann S, et al. Pregnancy loss and risk of cardiovascular disease: a prospective population-based cohort study (EPIC-Heidelberg). Heart 2011;97:49-54.

11. Kaandorp SP, Goddijn M, van der Post JA, et al. Aspirin plus heparin or aspirin alone in women with recurrent miscarriage. N Engl J Med 2010;362:1586-96.

12. Clark P, Walker ID, Langhorne P, et al. SPIN (Scottish Pregnancy Intervention) study: a multicenter, randomized controlled trial of low-molecular-weight heparin and low-dose aspirin in women with recurrent miscarriage. Blood 2010;115:4162-7.

13. Visser J, Ulander VM, Helmerhorst FM, et al. Thromboprophylaxis for recurrent miscarriage in women with or without thrombophilia. HABENOX: a randomised multicentre trial. Thromb Haemost 2011;105:295-301.

14. Pasquier E, de Saint Martin L, Bohec C, et al. Enoxaparin for prevention of unexplained recurrent miscarriage: a multicenter randomized double-blind placebo-controlled trial. Blood 2015;125:2200-5.

15. Rodger MA, Hague WM, Kingdom J, et al. Antepartum dalteparin versus no antepartum dalteparin for the prevention of pregnancy complications in pregnant women with thrombophilia (TIPPS): a multinational open-label randomised trial. Lancet 2014;384:1673-83.

16. van den Boogaard E, Vissenberg R, Land JA, et al. Significance of (sub)clinical thyroid dysfunction and thyroid autoimmunity before conception and in early pregnancy: a systematic review. Hum Reprod Update 2011;17:605-19.

17. Rai R, Regan L. Recurrent miscarriage. Lancet 2006;368:601-11.

18. Figueiredo AS, Schumacher A. The T helper type 17/regulatory T cell paradigm in pregnancy. Immunology 2016;148:13-21.

19. Kang X, Zhang X, Liu Z, et al. Excessive TLR9 signaling contributes to the pathogenesis of spontaneous abortion through impairment of Treg cell survival by activation of Caspase 8/3. Int Immunopharmacol 2015;29:285-92.

20. Porter TF, LaCoursiere Y, Scott JR. Immunotherapy for recurrent miscarriage. Cochrane Database Syst Rev 2006;2:CD000112.

21. Egerup P, Lindschou J, Gluud C, et al. The effects of intravenous immunoglobulins in women with recurrent miscarriages: a systematic review of randomised trials with meta-analyses and trial sequential analyses including individual patient data. PLOS One 2015; $10:$ e0141588

22. Tang AW, Alfirevic Z, Turner MA, et al. A feasibility trial of screening women with idiopathic recurrent miscarriage for high uterine natural killer cell density and randomizing to prednisolone or placebo when pregnant. Hum Reprod 2013;28:1743-52.

23. Liddell HS, Pattison NS, Zanderigo A. Recurrent miscarriage-outcome after supportive care in early pregnancy. Aust N Z J Obstet Gynaecol 1991;31:320-2.

24. Miyakis S, Lockshin MD, Atsumi T, et al. International consensus statement on an update of the classification criteria for definite antiphospholipid syndrome (APS). J Thromb Haemost 2006:4:295-306.

25. Laskin CA, Spitzer KA, Clark CA, et al. Low molecular weight heparin and aspirin for recurrent pregnancy loss: results from the randomized, controlled HepASA Trial. J Rheumatol 2009;36:279-87.

26. Wallace DJ. Does hydroxychloroquine sulfate prevent clot formation in systemic lupus erythematosus? Arthritis Rheum 1987;30:1435-6.

27. Johansson $\mathrm{E}$, Forsberg $\mathrm{K}$, Johnsson $\mathrm{H}$. Clinical and experimental evaluation of the thromboprophylactic effect of hydroxychloroquine sulfate after total hip replacement. Haemostasis 1981;10:89-96.

28. Johnson R, Charnley J. Hydroxychloroquine in prophylaxis of pulmonary embolism following hip arthroplasty. Clin Orthop Relat Res 1979:144:174-7.
29. Carter AE, Eban R. Prevention of postoperative deep venous thrombosis in legs by orally administered hydroxychloroquine sulphate. Br Med J 1974;3:94-5.

30. Wu TK, Tsapogas MJ, Jordan FR. Prophylaxis of deep venous thrombosis by hydroxychloroquine sulfate and heparin. Surg Gynecol Obstet 1977;145:714-8.

31. Ruiz-Irastorza G, Egurbide MV, Pijoan JI, et al. Effect of antimalarials on thrombosis and survival in patients with systemic lupus erythematosus. Lupus 2006;15:577-83.

32. Tektonidou MG, Laskari K, Panagiotakos DB, et al. Risk factors for thrombosis and primary thrombosis prevention in patients with systemic lupus erythematosus with or without antiphospholipid antibodies. Arthritis Rheum 2009;61:29-36.

33. Jung $\mathrm{H}$, Bobba R, Su J, et al. The protective effect of antimalarial drugs on thrombovascular events in systemic lupus erythematosus. Arthritis Rheum 2010;62:863-8.

34. Edwards MH, Pierangeli S, Liu X, et al. Hydroxychloroquine reverses thrombogenic properties of antiphospholipid antibodies in mice. Circulation 1997;96:4380-4.

35. Schmidt-Tanguy A, Voswinkel J, Henrion D, et al. Antithrombotic effects of hydroxychloroquine in primary antiphospholipid syndrome patients. J Thromb Haemost 2013;11:n/a-9.

36. Madow BP. Use of antimalarial drugs as "desludging" agents in vascular disease processes. J Am Med Assoc 1960;172:1630-3.

37. Ernst $E$, Rose $M$, Lee R. Modification of transoperative changes in blood fluidity by hydroxychloroquine: a possible explanation for the drug's antithrombotic effect. Pharmatherapeutica 1984;4:48-52.

38. Carter AE, Eban R, Perrett RD. Prevention of postoperative deep venous thrombosis and pulmonary embolism. Br Med $J$ 1971;1:312-4.

39. Espinola RG, Pierangeli SS, Gharavi AE, et al. Hydroxychloroquine reverses platelet activation induced by human IgG antiphospholipid antibodies. Thromb Haemost 2002:87:518-22.

40. Rand JH, Wu XX, Quinn AS, et al. Hydroxychloroquine directly reduces the binding of antiphospholipid antibody-beta2glycoprotein I complexes to phospholipid bilayers. Blood 2008;112:1687-95.

41. Gerstein HC, Thorpe KE, Taylor DW, et al. The effectiveness of hydroxychloroquine in patients with type 2 diabetes mellitus who are refractory to sulfonylureas--a randomized trial. Diabetes Res Clin Pract 2002;55:209-19.

42. Petri M, Lakatta C, Magder L, et al. Effect of prednisone and hydroxychloroquine on coronary artery disease risk factors in systemic lupus erythematosus: a longitudinal data analysis. Am $J$ Med 1994;96:254-9.

43. Virdis A, Tani C, Duranti E, et al. Early treatment with hydroxychloroquine prevents the development of endothelial dysfunction in a murine model of systemic lupus erythematosus. Arthritis Res Ther 2015;17:277.

44. Le NT, Takei Y, Izawa-Ishizawa Y, et al. Identification of activators of ERK5 transcriptional activity by high-throughput screening and the role of endothelial ERK5 in vasoprotective effects induced by statins and antimalarial agents. J Immunol 2014;193:3803-15.

45. Lafyatis R, York M, Marshak-Rothstein A. Antimalarial agents: closing the gate on Toll-like receptors? Arthritis Rheum 2006;54:3068-70.

46. Kyburz D, Brentano F, Gay S. Mode of action of hydroxychloroquine in RA-evidence of an inhibitory effect on toll-like receptor signaling. Nat Clin Pract Rheumatol 2006;2:458-9.

47. Kuznik A, Bencina M, Svajger U, et al. Mechanism of endosomal TLR inhibition by antimalarial drugs and imidazoquinolines. $J$ Immunol 2011;186:4794-804.

48. Bygbjerg IC, Svenson M, Theander TG, et al. Effect of antimalarial drugs on stimulation and interleukin 2 production of human lymphocytes. Int J Immunopharmacol 1987;9:513-9.

49. van den Borne BE, Dijkmans BA, de Rooij $\mathrm{HH}$, et al. Chloroquine and hydroxychloroquine equally affect tumor necrosis factor-alpha, interleukin 6 , and interferon-gamma production by peripheral blood mononuclear cells. J Rheumatol 1997;24:55-60.

50. Weber SM, Levitz SM. Chloroquine interferes with lipopolysaccharide-induced TNF-alpha gene expression by a nonlysosomotropic mechanism. J Immunol 2000;165:1534-40.

51. Broder A, Putterman C. Hydroxychloroquine use is associated with lower odds of persistently positive antiphospholipid antibodies and/or lupus anticoagulant in systemic lupus erythematosus. $J$ Rheumatol 2013;40:30-3.

52. Raschi E, Borghi MO, Grossi C, et al. Toll-like receptors: another player in the pathogenesis of the anti-phospholipid syndrome. Lupus 2008;17:938-43.

53. Döring $\mathrm{Y}$, Hurst J, Lorenz $\mathrm{M}$, et al. Human antiphospholipid antibodies induce TNFalpha in monocytes via Toll-like receptor 8. Immunobiology 2010;215:230-41. 
54. Berman J, Girardi G, Salmon JE. TNF-alpha is a critical effector and a target for therapy in antiphospholipid antibody-induced pregnancy loss. J Immunol 2005;174:485-90.

55. Kaplan YC, Ozsarfati J, Nickel C, et al. Reproductive outcomes following hydroxychloroquine use for autoimmune diseases: a systematic review and meta-analysis. Br J Clin Pharmacol 2016;81:835-48.

56. Götestam Skorpen C, Hoeltzenbein M, Tincani A, et al. The EULAR points to consider for use of antirheumatic drugs before pregnancy, and during pregnancy and lactation. Ann Rheum Dis 2016;75:795-810.
57. Tarfaoui N, Autret-Leca E, Mazjoub S, et al. [Hydroxychloroquine during pregnancy: a review of retinal toxicity in the newborns]. Therapie 2013;68:43-7.

58. Brigham SA, Conlon C, Farquharson RG. A longitudinal study of pregnancy outcome following idiopathic recurrent miscarriage. Hum Reprod 1999;14:2868-71.

59. Mekinian A, Lazzaroni MG, Kuzenko A, et al. The efficacy of hydroxychloroquine for obstetrical outcome in anti-phospholipid syndrome: data from a european multicenter retrospective study. Autoimmun Rev 2015;14:498-502. 